\title{
Time-Resolved Luminescence Detection and Imaging Promises a Bright Future
}

Xuedong Song and Stephen Quirk*

Global Director of Research and Engineering, Kimberly-Clark Corporation, USA

\section{Luminescence Detection Methods}

Fluorescence/phosphorescence (referred in this article simply as luminescence) based-detections arguably are the most widely used detection technique for biological assays, imaging, and sensing. This is primarily due to its high detection sensitivity and the commercial availability of a large number of fluorescence probes [1-3]. Key to the usefulness of luminescence methods is the ability to separate the luminescence signal of interest from the background. Such background includes scattered excitation photons and autofluorescence of sample matrices and substrates and it is often several orders of magnitude higher than the signal luminescence of interest. This low luminescence signal intensity relative to background sometimes presents a huge challenge when high detection sensitivity is required for the application at hand. Conventional luminescence detection technique uses wavelength differences to separate the luminescence of interest from background photons through optical filters or monochromators. Wavelength-based separation techniques sometimes face significant hurdles because most of the widely used luminescence probes have a very small Stokes shift and a broad luminescence spectrum. Hence, the complete elimination of the much stronger scattered excitation background is almost impossible. Complicating the issue further is the fact that the auto fluorescence from complex biological sample matrices and substrates sometimes overlaps with the luminescence of the probe. This makes it impossible to completely reject the background and results in a lower signal/noise ratio and therefore lower detection sensitivity.

An alternative technique for separating the luminescence signal from background is to take advantage of the lifetime difference between the fluorescence of the probe and the background photon [4]. The scattered excitation and other background photons are generally short-lived and decay rapidly. If the probe luminescence lifetime is much longer, the photon counting/measurement can start only after all the background and excitation photons disappear. The cycle can be repeated and the time-delayed luminescence signal can be summed. Because of the lower noise level, time-resolved luminescence (TRL) detection techniques can achieve more than two orders of magnitude higher detection sensitivity than the conventional luminescence detection techniques. In addition, the TRL detection technique needs only low-cost electronic components to achieve the luminescence signal separation and often doesn't need to use expensive band-pass optical filters or mono chromators. Furthermore, TRL detection techniques allow possible transmission detection of the luminescence signal that can collect the luminescence more effectively by placing the detector close to the probe. Transmission measurement mode also makes it possible to construct miniaturized detectors.

\section{Probes}

Although TRL detection promises many advantages over conventional luminescence detection techniques, lack of a wide variety of useful probes has significantly hindered its wide adoption. In addition to the desirable features of conventional luminescence probes such as strong absorption in the visible or near IR region and high luminescence quantum yield, suitable probes for time-resolved

luminescence detection need to have a luminescence lifetime of $>1 \mu \mathrm{s}$ to realize the theoretical benefits of the technique. The long lifetime requirement is critical for all the background to be completely rejected and also if low cost electronics are to be used to achieve the separation.

A review of the scientific literature has revealed only two major classes of compounds that are suitable for practical TRL-based detection. The most widely used class of probe is lanthanide-based chelates, primarily europium-chelates [5]. The lanthanide-based chelates have very strong absorption around 330-375 nm and emit strong fluorescence at $\sim 615 \mathrm{~nm}$ with a $\sim 500 \mu$ s lifetime. Their large Stokes shift $(\sim 250 \mathrm{~nm})$ is also beneficial. However, the requirement for a UV excitation light source is a significant drawback. More recently, new classes of lanthanide chelates have been developed that have a better excitation profile near or in the blue region. These chelates can be excited by low cost light sources (390 to $420 \mathrm{~nm}$ LEDs).

A second class of suitable probes is noble metal chelates, specifically platinum and palladium chelates, which emit strong phosphorescence at $650 \mathrm{~nm}$ with a long lifetime $(500 \mu \mathrm{s})$ and exhibit a large Stokes shift $[6,7]$. Those chelates can be easily excited by low cost, commercially available, UV LEDs or blue LEDs (390 to $420 \mathrm{~nm}$ ). However, the phosphorescence nature of platinum and palladium chelates' luminescence makes it vulnerable to oxygen quenching. This dictates that signal will be measured in an oxygen-free environment. This inconvenience has prevented realization of their commercial potential.

Although the association constants of the lanthanide ions and their common ligands (as well as the noble metal ions and their cyclic ligands) are very high, the dissociation of these metal chelates is still possible. Such disassociation will significantly diminish the luminescence intensity, which will result in lower detection sensitivity when the concentration of the chelates in the assay is very low. The chelate dissociation issue at low concentration can be overcome by using particles that encapsulate those luminescence molecules. The polymeric matrix cannot just only prevent the chelates from dissociating, but would also provide signal amplification because one particle can contain a large number of chelates. Recently, both micro- and nano-particles have been developed and commercialized to encapsulate those chelates to achieve signal amplification, and at the same time, to prevent the chelates from dissociating at very low concentration [8]. Europium-based nanoparticles with various surface

*Corresponding author: Stephen Quirk, Global Director of Research and Engineering, Kimberly-Clark Corporation, USA; Tel: 770-587-8541; Fax: 770-5877758; E-mail: squirk@kcc.com

Received November 08, 2012; Accepted November 09, 2012; Published November 10, 2012

Citation: Song X, Quirk S (2012) Time-Resolved Luminescence Detection and Imaging Promises a Bright Future. J Biosens Bioelectron 3:e115. doi:10.4172/2155$6210.1000 \mathrm{e} 115$

Copyright: ( $) 2012$ Song X, et al. This is an open-access article distributed under the terms of the Creative Commons Attribution License, which permits unrestricted use, distribution, and reproduction in any medium, provided the original author and source are credited. 
functional groups are commercially available from a number of vendors. The surface functional groups allow covalent attachment of various biological molecules such as antibodies to the particle surface. For phosphorescent nanoparticles, the benefit of encapsulation also includes minimization of oxygen quenching under ambient conditions so that an oxygen-free measurement environment is no longer a constraint. Several types of polymers have been used to encapsulate phosphorescent molecules to reduce the oxygen quenching, including polymer styrene, polyacrynitrile, silica, and halogen-containing polymers $[9,10]$. Polystyrene-based phosphorescent particles have proven less than ideal because of relatively low intensity and short lifetimes. This is due to the relatively high level of oxygen permeability and the resulting phosphorescence quenching.

\section{Applications}

\section{Time-resolved luminescence assays}

A large number of assays have been developed and reported in the literature using TRL detection [11]. Applications cover a wide variety of analytes and reactions, including ligand-receptor binding interactions, detection of small molecules, nucleic acid hybridizations, and enzymes assays [12-14]. In general, the probes used for TRL homogenous assays are metal chelates, particularly europium chelates with various ligands. Those chelates are modified to be water soluble and often covalently attached to molecules of interest to form a conjugate. Not only are the homogeneous TRL assays of academic interest, they are also of high commercial interest. A number of commercial assay products have been on the market that provides increased performance for high through drug screening and medical diagnostics.

\section{Time-resolved luminescence imaging}

A less explored application of TRL-based detection is in the field of imaging [15-17]. Two types of TR luminescence imaging methods; frequency domain and time domain, have been reported. Emerging research should find a wide range of potential applications by discriminating and mapping different fluorophores. This will lead to a better understanding of cells and tissue micro environments. This in turn will lead to improved diagnosis of certain diseases and further understanding of cellular and tissue molecular transport.

\section{Time-resolved luminescent lateral flow assays}

Recently TRL detection has been integrated into a low cost and user-friendly lateral flow chromatographic assay platform to provide a powerful point-of-care (POC) and over-the-counter (OTC) detection platform $[18,19]$. Two new types of luminescent nanoparticles with long luminescence lifetimes have made it practical to develop the technology platform. One type of nanoparticle uses halogen-containing polymers that encapsulate phosphorescent molecules (platinum and palladium chelates) to minimize the oxygen dependent quenching of phosphorescence [20]. The particles can be easily excited by low-cost LEDs from 390 to $410 \mathrm{~nm}$ to emit strong phosphorescence at $650 \mathrm{~nm}$ with about a $100 \mu$ s lifetime coupled with a large Stokes shift under ambient conditions. A second type of nanoparticle encapsulates a lanthanide chelate that has a strong absorption peak around $415 \mathrm{~nm}$ and emits strong fluorescence at $615 \mathrm{~nm}$ with a fluorescence lifetime of approximately $500 \mu$ s. Unlike commercial nanoparticles with long luminescence lifetimes that can be effectively excited only by UV light, the new particles can be efficiently excited from 400 to $430 \mathrm{~nm}$. This feature of excitation by visible light is tremendously advantageous for cost, miniaturization and elimination of interference from complex biological samples and platform matrices.

\section{Key challenges/opportunities}

Although TRL detection techniques have been widely utilized for a number of applications, the limited options of compatible and truly functional probes have remained a major hurdle for wider adoption. Almost all the commercial probes suitable for TRL detection for biological assays and medical diagnostics can be effectively excited only by UV which as noted above, is not ideal. Most biological sample matrices and substrates have significant absorption in the UV, which can interfere with the excitation of those probes. UV excitation is also not amenable for biological imaging due to potentially damaging live cells. Moreover, the UV light resource is generally more expensive, less powerful, somewhat hazardous, and is not very appropriate for POC and OTC products. Therefore, there are tremendous needs to develop new molecular probes with suitable spectral profiles, preferably excitable at $>450 \mathrm{~nm}$. Another challenge is that most of commercial instruments related to luminescence measurements don't have capability of measuring time-resolved luminescence. However, this challenge could be easily resolved if suitable probes with excitable wavelength of $>450 \mathrm{~nm}$ were widely available. The same is true for TRL imaging. A low cost CCD camera coupled with a time-gating capability will be another key technological innovation of TRL imaging with applications for biochip-based multiplexing systems such as nucleic acid or protein microarrays. Another area of opportunity is to use TRL for flow cytometric analysis of cells. Finally a significant opportunity exists for developing detection systems and assays that simultaneously use wavelength-based separation and lifetime-based separation methods to accomplish analyte multiplexing. In combining conventional and TRL detection techniques, it is possible to develop particle arrays that can increase throughput exponentially. Similarly a TRL imaging system utilizing the two detection methods can trace the behaviors of a number of targets on the same spot and at the same time. However, all of this innovation and new sensing modalities must begin with the continued development of novel and differentially functional time-resolved luminescent molecular probes and nanoparticles.

\section{References}

1. Hanson GT, Hanson BJ (2008) Fluorescent Probes for Cellular Assays. Comb Chem High Throughput Screen 11: 505-513.

2. Lakowicz JR (1999) Principles of Fluorescence Spectroscopy, (3rdedn). Plenum Publishers.

3. Scholl PF, Bargeron CB, Phillips TE, Wong T, Abubaker S, et al. (2000) Immunoaffinity based phosphorescent sensor platform for the detection of bacterial spores. Proceedings of SPIE 3913: 204-214.

4. Dickson EF, Pollak A, Diamandis EP (1995) Ultrasensitive bioanalytical assays using time-resolved fluorescence detection. Pharmacol Ther 66: 207-235.

5. Handl HL, Gillies RJ (2005) Lanthanide-based luminescent assyas for ligandreceptor interactions. Life Sci 77: 361-371.

6. Burke M, O'Sullivan PJ, Soini AE, Berney H, Papkovsky DB (2003) Evaluation of the phosphorescent palladium(II)-coproporphyrin labels in separation-free hybridization assays. Anal Biochem 320: 273-280.

7. O'Riordan T, Soini AE, Soini JT, Rapkovsky DB (2002) Performance evaluation of the phosphorescent porphyrin label: solid-phase immunoassay of alphafetoprotein. Anal Chem 74: 5845-5850.

8. Cummins CM, Koivunen ME, Stephanian A, Gee SJ, Hammock BD, et al. (2006) Application of europium(III) chelate-dyed nanoparticle labels in a competitive atrazine fluoroimmunoassay on an ITO waveguide. Biosens Bioelectron 21 : 1077-1085.

9. Kürner JM., Klimant I, Krause C, Pringsheim E, Wolfbeis OS (2001) A new type of phosphorescent nanospheres for use in advanced time-resolved multiplexed bioassays. Anal Biochem 297: 32-41.

10. Kürner JM, Klimant I, Krause C, Preu H, Kunz W, et al. (2001) Inert 
Citation: Song X, Quirk S (2012) Time-Resolved Luminescence Detection and Imaging Promises a Bright Future. J Biosens Bioelectron 3:e115. doi:10.4172/2155-6210.1000e115

Page 3 of 3

phosphorescent nanospheres as markers for optical assays. Bioconjug Chem 12: 883-889.

11. Diamandis EP (1988) Immunoassays with time-resolved fluorescence spectroscopy: principles and applications. Clin Biochem 21: 139-150.

12. Degorce F, Card A, Soh S, Trinquet E, Knapik GP, et al. (2009) HTRF: A technology tailored for drug discovery: a review of theoretical aspects and recent applications. Curr Chem Genomics 3: 22-32.

13. Claret E, Quled-Diaf J, Seguin P (2003) Homogeneous time-resolved fluorescence assay to measure histamine release. Comb Chem High Throughput Screen 6: 789-794.

14. O'Shea D, O'Riordan TC, O'Sullivan PJ, Papkovsky DB (2007) Homogeneous time-resolved fluorescence assays for the detection of activity and inhibition of phosphatase enzymes employing phosphorescently labeled peptide substrates. Anal Chim Acta 583: 349-356.
15. Cubeddu R, Comelli D, D’Andrea C, Taroni P, Valentini G (2002) Time-resolved fluorescence imaging in biology and medicine. J Phys D Appl Phys 35: R61.

16. Hassler K, Anhut T, Lasser T (2005) Time-resolved Hadamard fluorescence imaging. Appl Opt 44: 7564-7572.

17. Herman P, Lin H, Lakowicz JR (2003) Lifetime-based imaging. Tuan Vo-Dinh (Editor-in-chief) Biomedical Photonics Handbook, CRC Press.

18. Song X (2009) Lateral Flow Immunoassay Using Time-Resolved Luminescence Detection. IVD Technologies 15: 31-37.

19. Song X, Knotts M (2008) Time-Resolved Luminescent Lateral Flow Assay Technology. Anal Chim Acta 626: 186-192.

20. Song X, Huang L, Wu B (2008) Bright and monodispersed phosphorescent particles and their applications for biological assays. Anal Chem 80: 5501-5507. 